\title{
ACREDITACIÓN INSTITUCIONAL EN LA UNIVERSIDAD DE LA FRONTERA. DESAFÍOS DESDE LA EXPERIENCIA
}

Sergio Bravo* 


\section{RESUMEN}

La Universidad de La Frontera (UFRO) culminó la etapa de autoevaluación institucional en el contexto de la búsqueda de su acreditación con la Comisión Nacional de Acreditación de Pregrado (CNAP). La ocasión sirvió no sólo para reflexionar críticamente acerca de los procesos de regulación de calidad, sino para aventurarse a la apertura de posibilidades de desarrollo institucional. En esa perspectiva, este artículo considera tres secciones: la mirada retrospectiva, que da cuenta de los grados crecientes de complejización de la UFRO desde su fundación; el análisis de la experiencia, que, en categorías analíticas gruesas, muestra los resultados de la autoevaluación institucional; y visión de futuro, análisis prospectivo de las oportunidades de diseño de nuevas políticas y estrategias de desarrollo que, en un marco de participación académica amplia, le permitan posicionarse en un lugar relevante dentro del contexto de la educación superior chilena.

\section{ABSTRACT}

The Universidad de La Frontera (UFRO) completed the stage of institutional self-assessment as part of its search for accreditation with the National Commission for Undergraduate Accreditation (CNAP). This instance was an occasion for the University to reflect critically not only on its quality regulation processes but also to undertake to open up new possibilities of institutional development. In this perspective, this paper is divided into three sections: a retrospective analysis that describes the increasing degrees of complexity of the UFRO since the time it was established; an analysis of the experience that -in broad analytical categories- shows the outcome of institutional selfassessment; and the view for the future, an analysis of prospects for designing new opportunities for new development policies and strategies that -in a framework of broad academic involvement- will allow the UFRO to achieve a significant position in the context of Chile's higher education system. 


\section{ACREDITACIÓN INSTITUCIONAL EN LA UNIVERSIDAD DE LA FRONTERA. DESAFÍOS DESDE LA EXPERIENCIA}

La mirada retrospectiva

El origen de la Universidad de La Frontera se remonta al año 1948, en que el Grado de Técnicos de la Escuela Industrial de Temuco pasó a formar parte de la Universidad Técnica del Estado, cuyo desarrollo permitió que, en 1960, ya contara con dos carreras de nivel universitario. En ese año se creó en esta ciudad el Colegio Universitario Regional de la Universidad de Chile.

Para esa época, Temuco contaba con 80 mil habitantes y las dos provincias que hoy conforman la IX Región (Malleco y Cautín) sumaban 600 mil. La comunidad local tenía hacía tiempo el anhelo de contar con un centro formador que diera respuesta a las necesidades de desarrollo de la región y a los egresados de la educación secundaria, quienes, en esos tiempos, se veían obligados a emigrar hacia otras grandes ciudades para seguir una carrera profesional (Pinto, 2002).

En este contexto nace la Universidad de La Frontera, creada en marzo del año 1981 como Universidad Estatal Derivada, a partir de la fusión de las sedes de las Universidades de Chile y Técnica del Estado en Temuco. Se crea con la misión de dedicarse a la enseñanza y al cultivo superior de las artes, las letras y las ciencias, contribuyendo de esta manera al desarrollo de la IX Región.

Con sus 23 años de vida institucional, ha logrado un notable desarrollo en diversas áreas. Ha mejorado de manera sustantiva la calificación del recurso humano, ha aumentado y diversificado sus actividades académicas, ha potenciado sus programas de formación, generando grupos consolidados en investigación y constituyéndose en un referente importante para el contexto regional en el que está inserta. A ello ha contribuido una situación financiera saludable, que 
ha permitido el notable incremento de su patrimonio, el crecimiento y modernización de la infraestructura y del equipamiento que sirve de soporte a todas las actividades de la institución, y, en general, el mejoramiento de las condiciones laborales.

Las áreas deficitarias y las necesidades de mejoramiento son muchas y muy variadas. Aún persisten, en algunas áreas del conocimiento, modelos muy tradicionales acerca del ser y hacer de la universidad, lo que determina una visión bastante enciclopedista de la enseñanza, con poca consideración de los procesos de aprendizaje, con una sobrevaloración de la investigación pura y una desvinculación con el entorno social, productivo y económico. El proceso de desarrollo de una universidad tan joven como la UFRO recién ha comenzado. El cambio cultural que se requiere para dar el salto cualitativo, necesario para transformarse en una universidad compleja y multifuncional, requiere de un compromiso activo de todos los integrantes de la comunidad universitaria y de una gran capacidad para, por una parte, aunar las visiones y formas de hacer universidad y, por otra, concretar su desarrollo en forma sostenida ${ }^{1}$.

Este cuadro general de fortalezas y oportunidades, y de debilidades y amenazas, se intensifica en la última década, que marcó a su vez, para las universidades chilenas, una época de profundos retos. Se les han hecho demandas, provocando en ellas efectos diversos pero, sobre todo, desconcierto y una "crisis de identidad"(Zurita, 1998). Al interior de las universidades estatales han ocurrido procesos que las han desafiado en lo que concierne a tareas fundamentales, como la producción del conocimiento y la capacidad de constituirse, realmente, en instancia de formación de las generaciones futuras, en los distintos campos del saber y en relación con los requerimientos actuales. Nuestra universidad no ha estado ajena a estas demandas y desafíos.

Las políticas y estrategias desde las cuales se regula hoy a la universidad han ido adquiriendo su fuerza y viabilidad en el poder de instrumentos que afectan la naturaleza, contenido y organización del trabajo universitario. Licenciamiento, acreditación de programas y la puesta en el escenario público del debate acerca de las "Orientaciones Para una Política de Aseguramiento de la Calidad en Chile" son instrumentos que han estado constituyéndose, paulatina pero

1 Informe de Autoevaluación Institucional. Universidad de La Frontera, mayo de 2004. 
efectivamente, en normas abstractas asociadas a procedimientos estandarizados de aplicación general. Estos instrumentos proyectan sus exigencias como tareas a ser autoasumidas por las propias instituciones, para estar en condiciones de responder adecuadamente a los desafíos que plantean sus nuevas realidades (Herrera, 2002).

La misión de la UFRO, en cuanto pública y regional, establece como imperativo su articulación con las demandas sociales y necesidades de desarrollo de la comunidad regional y la sociedad nacional. La IX Región, de acuerdo a todos los estándares y parámetros, es una de las más deprimidas del país en términos de su desarrollo humano. Ha sido vocación de la universidad transformarse en un agente de reflexión crítica y en elaboradora de propuestas que impacten positivamente en los indicadores regionales.

Las nuevas exigencias que se le hacen a la universidad, así como los imperativos que emanan de su visión y misión (origen de la crisis y desconcierto), las hemos interpretado más como oportunidades y desafíos que como amenazas; como la apertura a la posibilidad de construir un accionar en que la autonomía se ubique como elemento estratégico de nuestro desempeño, como expresión de nuestras particulares formas de organización y como una forma esencial de búsqueda de identidad. Hoy se encuentra inmersa en un espacio que la obliga a competir para acceder a una posición institucional que ayer podía garantizar mediante las relaciones corporativas con el Estado de las dos instituciones que le dieron origen. Lo anterior, sin embargo, requiere de una forma de gobierno y administración que generen, a través de la construcción de nuevas identidades, cambios en la operación de las actividades consustanciales a nuestro quehacer universitario.

En este nuevo escenario, debió confrontar los desafíos que significaba hacerse cargo, primero de sí misma y luego -con un fuerte sesgo de responsabilidad social- de las demandas que emergen de una ecología social cada vez más compleja, dinámica y competitiva. Ello requería de una redefinición de su proyecto institucional para la recreación de su identidad, a partir de su historia particular y de las condiciones locales concretas donde realiza su acción. Sólo así los cambios operados proyectarían una diferenciación en la educación superior, según la visión y la misión institucionales que deben reflejarse en el tipo de programas que ofrece, en la forma que atiende a sus 
beneficiarios y en la relevancia y pertinencia de la construcción del conocimiento a través de la investigación.

Debió hacerse cargo entonces de un imperativo nuevo, que se relaciona con asumir un tipo nuevo de autonomía: aquella consistente con la responsabilidad sobre nuestra propia conducción y desempeño y que, en términos prácticos, se ubica en los campos de acción que garanticen la regulación a distancia que ejerce el Estado. Dicho en términos operativos, requería de una nueva "gobernabilidad institucional, es decir, [de] la capacidad de las universidades para conducirse como comunidades capaces de cumplir cabalmente con sus finalidades autoimpuestas (Ibarra, 2001)". Ella supone, entre otros aspectos, la capacidad de consulta, diálogo y decisión, manejo eficiente de recursos y programas, diseño de sistemas y procedimientos de y para la acción, y delimitación de proyectos institucionales legítimos que otorguen identidad y faciliten el consenso en el marco de redes de colaboración.

Es frente a este imperativo, y a la necesidad de regular la calidad de su quehacer para enfrentar mejor los desafíos esbozados, que la Universidad de La Frontera tomó la decisión de aceptar la invitación cursada por la Comisión Nacional de Acreditación de Pregrado (CNAP), en mayo del año 2003. Con anterioridad, la institución se encontraba realizando actividades preparatorias para el desarrollo de un proceso interno, conducente a generar un modelo de evaluación institucional, en el marco del Plan de Trabajo 2003 de la Dirección de Autoevaluación y Acreditación de la universidad. Las áreas de evaluación obligatorias para todas las instituciones participantes (Gestión Estratégica y Docencia de Pregrado) fueron complementadas con Investigación, decisión que se tomó a partir de un análisis realizado por el equipo directivo, que consideró la necesidad de evaluarla porque se trata de un factor clave para el desarrollo institucional y, por otra parte, porque se puede beneficiar de manera significativa de una evaluación profunda de sus políticas, programas, procedimientos y resultados.

\section{El análisis de la experiencia}

No quisiera dar cuenta detallada de lo que significó la experiencia del proceso de autoevaluación institucional, sino, más bien, referirme a categorías cualitativas gruesas que emergieron de la contrastación de 
nuestras orientaciones de políticas con lo que podemos dar cuenta acerca de lo que hacemos.

En la actualidad, la institución cuenta con orientaciones de políticas para las funciones universitarias básicas, a lo que habría que agregar las políticas de desarrollo estudiantil y de perfeccionamiento y posgrado. Sin embargo, era necesario iniciar un proceso reflexivo en términos de lo deseable, para enriquecer estas políticas en función de un horizonte de desarrollo a mediano plazo. La acreditación institucional ofreció esa oportunidad. De ese modo, por ejemplo, la noción de "elite académica", que conforma la imagen que la mayoría de la gente posee de la universidad en términos generales, se relaciona, obviamente, con profesores y estudiantes de excelencia capaces de realizar docencia e investigación sofisticada, teórica y/o metodológicamente. Sería ilusorio pensar que ésta característica se hace extensiva a todo el quehacer universitario en la UFRO, pero debe ser exigible (una condición sine-qua-non) para los programas de formación profesional, el posgrado y el financiamiento de la investigación.

En esa perspectiva, podemos señalar que la universidad se encuentra posicionada en un lugar relevante dentro del concierto de las pertenecientes al Consejo de Rectores. En términos de la función docente, se han hecho variados esfuerzos, tanto en el plano de la elaboración de políticas como en el del desarrollo cualitativo de la docencia, que se han trasuntado en innovaciones curriculares y en la elaboración de instrumentos de evaluación. En el plano de la investigación, se encuentra ubicada en el $7^{\circ}$ lugar nacional de producción científica, resultado de orientaciones y de esfuerzos individuales y grupales, que han sido acompañados por la creación de institutos interdisciplinarios y centros de investigación y desarrollo.

Sin embargo, tan auspiciosa ubicación relativa es frágil si se considera que, tanto los grupos de investigación como los esfuerzos innovativos en docencia, reflejan una heterogeneidad, en el sentido en que han comprometido sólo a una parte minoritaria del universo de los académicos. Ello producto de formas de planeamiento centralizadas que encauzan las potencialidades de grupos de elite, pero que, por su naturaleza, se muestran inapropiadas para convocar al grueso de los académicos a la gestión y desarrollo de ideas y productos. 
Cualesquiera sean las interpretaciones acerca de la situación actual de la Universidad de la Frontera, pareciera existir consenso entre los miembros de esta comunidad académica acerca de que uno los mayores desafíos para su evolución futura se relaciona tanto con la acreditación institucional como con la de sus procesos de formación de profesionales. Estos desafíos contienen horizontes que se deben alcanzar en el corto y mediano plazo y que requieren de la acción concertada de los académicos miembros de los departamentos y facultades para alcanzarlos. La elaboración de estándares, la acreditación de las carreras, la constitución de un espacio institucional que contenga a las carreras (escuelas, por ejemplo) constituyen desafíos urgentes de abordar. La autoevaluación institucional nos ha dado mayores luces para orientar nuestra acción, en la medida en que la hemos asumido en una perspectiva más general que denominamos "Evaluación y Desarrollo" (E+D).

La estrategia de E+D nos brinda a todos la oportunidad de involucrarnos en el proceso de decidir lo que debemos implementar, quiénes asumen diferentes responsabilidades y la calendarización más eficiente y efectiva de las actividades. Además, a través de una aproximación rigurosa, sistemática pero a la vez práctica, nos permite la formalización, como universidad, de estándares de calidad y mecanismos de desarrollo académico para responder a las demandas externas e internas. Más aún, nos capacita para diseñar las estrategias mediante las cuales una unidad académica pueda operar como una organización de y para el aprendizaje.

Dentro de las urgencias para el desarrollo estratégico de la UFRO, detectadas como resultado del proceso de autoevaluación, emergen cuatro fundamentales: la autonomía y búsqueda de identidad, el aumento de la productividad académica, el incentivo e implementación de programas de posgrado de excelencia, y el desarrollo cualitativo de la docencia para atender más efectivamente los requerimientos sociales y laborales, y completar los procesos de acreditación de los programas que conforman su oferta docente. En esta perspectiva, el diseño de un proceso participativo de planificación es un aspecto clave para alcanzar los propósitos enunciados. La autoevaluación en la perspectiva E+D se transforma en una herramienta fundamental para este proceso.

La implementación del proceso que se inauguró con la autoevaluación institucional significó un cambio radical en los modos 
y prácticas de la gestión y planeamiento de la actividad académica y docente. En tal sentido, se espera, con el diseño de esta estrategia de $\mathrm{E}+\mathrm{D}$, sentar las bases para el desarrollo de una cultura de evaluación y compromiso institucional. Ello significa establecer un plan para la evaluación sistemática de las carreras y la productividad académica que sirva como sustento para la redefinición de la misión institucional, en función de la participación y compromiso de los actores involucrados, y que tiendan a la validación externa en el contexto del incremento de la calidad y vinculación con los desafíos del desarrollo regional y nacional. En esa perspectiva, el acercarse a un modelo de planificación en la base abre las posibilidades de enfrentar la apatía y esfuerzos aislados, no sistemáticos, que caracterizan a cierta actividad académica en la universidad. Se espera catalizar los procesos de reflexión crítica y de compromiso de los departamentos y carreras para que se abran a la posibilidad de la innovación, el cambio y el aumento de la productividad académica.

Como hemos señalado, aun cuando la universidad cuenta con orientaciones generales de políticas para sus funciones académicas básicas y un Plan de Desarrollo marco, su gestación ha sido más bien cupular y no responde, verdaderamente, a definiciones políticas como "discurso de segundo orden", en el sentido que Fisher (1995) le asigna a la deliberación de políticas ${ }^{2}$. La alternativa de seguir con las mismas prácticas y formas de planeamiento pone en riesgo tanto el posicionamiento institucional alcanzado como la posibilidad de potenciar grupos, unidades y programas que no se han sentido

2 Fischer (1995) caracteriza la deliberación de políticas en cuatro niveles: verificación técnica, validación situacional, vindicación del sistema y elección social racional. El discurso de los dos primeros niveles se realiza en el contexto del sistema de valores prevaleciente en las organizaciones e instituciones sociales. La verificación técnica conlleva la evaluación de una política con el propósito de medir su efectividad empírica. En el segundo nivel, validación situacional, el análisis debiera determinar si los criterios usados para juzgar la política son válidos.

En los niveles más altos, que Fischer denota como discurso de segundo orden, la deliberación de políticas concierne a la justificación y aceptabilidad del sistema de valores que se adopta para juzgar la política, algunas veces llegando a lo profundo de las convicciones acerca del orden social preferido y modo de vida. El tercer nivel es el de la vindicación del sistema o argumentación política general, donde los tópicos generales giran en torno a la compatibilidad de la política examinada con los valores y propósitos generales y problemas de justicia distributiva, con una atención particular a los aspectos no considerados y a sus efectos colaterales. Y aún a un nivel mayor (el de la elección social racional o argumentación ideológica) se ubica el debate profundo, en instancias donde un cambio de vida y la adopción de ideales sociales radicalmente diferentes parecen necesarios para alcanzar ciertos propósitos. La gran preocupación de Fischer es llevar el debate de políticas a estos aspectos profundos, en oposición a mantenerse limitados a la adecuación de los diseños de políticas al nivel operacional de la verificación técnica. 
convocados por las políticas institucionales, debido a su carácter vertical que refuerza el componente de marginalidad. En este sentido, esta estrategia se plantea en el contexto de una suerte de "reencantamiento" institucional participativo. Para alcanzar tales propósitos se requiere de definiciones políticas y de una cultura organizacional que apoye al proceso. Quizás estas sean las corroboraciones más relevantes que nos dejó el proceso de autoevaluación, y su respectivo informe, que acabamos de culminar.

Visión de futuro: avances hacia la definición de políticas

En el nuevo escenario de regulación de la calidad de las universidades podemos caer en la tentación de que esta fase inicial (la de autoevaluación institucional), quede circunscrita al nivel más bajo de la argumentación acerca de políticas: el de la verificación técnica. Debemos esperar, por el contrario, discusiones acerca de, por ejemplo, el significado de conceptos tales como "estándar educacional", "excelencia" y "calidad académica". De ese modo, abriremos espacios para mayores reflexiones. Los procesos de acreditación no deben focalizarse sólo en procedimientos operacionales y en detalles de diseño, no dejando ningún espacio para la ambivalencia y la resignificación, sin mencionar el involucramiento de otros actores relevantes en la formación profesional, fuera de los académicos y el Estado.

Si operáramos sobre la premisa de que "sólo lo que es medible es lo que se hace o se interviene", estaríamos considerando los aspectos negativos o positivos más obvios de un proceso de acreditación. Su consecuencia, a largo plazo, sería una severa restricción de espacios para la reflexión, innovación y evolución en el campo de la construcción compartida de una nueva identidad. Debemos estar alertas porque el Estado no puede darse el lujo de ignorar los aspectos de eficiencia de cualquiera de sus políticas. En una segunda fase de una política de aseguramiento de la calidad creo que este argumento va a tomar un lugar prominente. Puede que éste todavía se dé en una primera instancia al nivel de verificación técnica, dentro del marco establecido para la acreditación institucional y de programas. Pero la cuestión a examinar en el futuro no es solamente si un programa o institución cumple con los estándares requeridos, independientemente de los costos asociados y de las premisas que subyacen a los procesos de planificación y formación. 
En suma, y para ponerlo de otro modo, la UFRO no sólo debe intentar resolver los problemas de aseguramiento de calidad propuestos por el Estado (a través de los procesos de acreditación), sino abocarse también a la búsqueda de su redefinición en consonancia con sus declaraciones de visión, misión y perfiles, a través de la construcción de una nueva gobernabilidad, de nuevos enfoques y modelos que garanticen su adecuado cumplimiento. En ese espacio, podremos enfrentar mejor los desafíos actuales y futuros.

Como complemento inseparable debemos agregar la necesidad de tener una universidad más eficiente, con mayor capacidad de respuesta a los requerimientos del desarrollo nacional, que asegure la equidad, en el sentido de brindar igualdad de oportunidades en el acceso y en el aprendizaje, y que diversifique la fuerza laboral en el contexto de la globalización, compatible con la necesidad de mantener el legado cultural de la nación. Estos son tópicos que emergen como telón de fondo a un debate más profundo acerca de la calidad de nuestro quehacer y que no pueden ser "resueltos" por un solo y perfecto esfuerzo de diseño cupular.

Por otra parte, el problema de la productividad en la UFRO urge a un cambio paradigmático, que suponga salirse de los cánones de su consideración en términos de la racionalidad estrictamente económica para aventurarnos en la de los productos de nuestra acción o práctica académica. Dicho de otro modo, el problema de la productividad se debe menos al aumento de los costos que a la baja en los productos de aprendizaje efectivo. Desde este enfoque, se deduce que el aumento de la productividad académica debería centrarse en el aumento de la producción de conocimientos (investigación) y de formas apropiadas de transferencia de conocimientos, para resolver los problemas de desarrollo de la sociedad a través de la adecuada articulación con el entorno y la formación profesional.

Lo anterior equivale a expresar que el avance en términos de productividad no puede alcanzarse completa o aún importantemente mediante el enfoque tradicional de reducir o abaratar los costos de los insumos -principalmente académicos, docentes y administrativos-, manteniendo constante (o aumentando) la matrícula. Tal punto de vista intenta resolver los problemas coyunturales de recursos insuficientes sacrificando los productos que realmente interesan: la calidad de los procesos de construcción y transferencia de 
conocimientos. Los avances significativos y sustentables en productividad académica deberían alcanzarse poniendo mayor atención a los beneficiarios: la sociedad y los estudiantes. Construcción de conocimientos y productividad en los aprendizajes relacionan los insumos académicos y administrativos no a la matrícula, sino a la calidad de lo que se construye y aprende; a la demostración madura de un cuerpo de conocimientos o habilidades. Cuando el objeto de reflexión crítica son los constructores de conocimiento, el aprendizaje y los aprendices, se abre un enorme potencial de posibilidades, no para hacer más de lo mismo a un menor costo, sino para mejorar la práctica académica en función de resultados que tengan impacto social, haciendo una contribución sustantiva al desarrollo regional y nacional.

Lo anterior constituye un conjunto de ideas-fuerza para incentivar el diálogo y la participación de la base académica en la perspectiva del diseño de políticas de desarrollo institucional como discurso de segundo orden. En esta perspectiva, es menester agregar que, cuando hablamos de "políticas", nos estamos refiriendo, principalmente, a un curso de acción público estable (pero modificable) destinado a un ajuste óptimo entre diferentes opciones, todas las cuales pueden alcanzarse en forma independiente, pero que se maximizan cuando se tratan en conjunto (Herrera, 2000).

Se debe señalar que la propuesta de políticas responde siempre más a preguntas prácticas que a teóricas. Las declaraciones acerca de lo que debemos hacer producto del análisis de nuestro proceso de autoevaluación constituye una ventaja en la perspectiva de que se abren las posibilidades de arribar a consensos en la acción, antes que tratar de concordar en las razones teóricas de por qué hacerlo, que se verifican en un dominio y lógicas diferentes. De ese modo, la propuesta de políticas se constituirá en líneas de acción, especificaciones del quehacer que se deberán plasmar en un nuevo Plan de Desarrollo Institucional, informado y participativo, primordialmente expresión de convicciones morales y estimaciones de valor.

Así, con el compromiso de todos y sin afanes protagónicos personalizados, con generosidad y esfuerzos compartidos, podremos avanzar en la constitución de una verdadera comunidad académica y de una nueva identidad para la universidad en tiempos complejos y de cambio. La autoevaluación y la acreditación son procesos que nos abren a esa posibilidad. 


\section{REFERENCIAS BiBLIOGRÁFICAS}

Fischer, F., Evaluating Public Policy, Ed. Nelson-Hall, Chicago, 1995.

Herrera, Ricardo, "Epistemologías Locales y Prácticas Pedagógicas de Formación Profesional", en Las Nuevas Demandas del Desempeño Profesional y Sus Implicancias Para la Docencia Universitaria, Centro Interuniversitario de Desarrollo, CINDA-MINEDUC, 2000.

Ibarra, Eduardo, "La Universidad y sus Dilemas. ¿De la Universidad Empresarial a la Gobernabilidad Participativa?", en Las Ciencias Sociales, Universidad y Sociedad. Temas Para Una Agenda de Postgrado. UNAM, México, 2001.

Informe de Autoevaluación Institucional. Universidad de La Frontera, Temuco, mayo de 2004.

Pinto, Jorge, Historia de la Universidad de La Frontera, Ediciones Universidad de La Frontera, Temuco, 2002.

Zurita, Reginaldo, "Crisis de Identidad en la Universidad Chilena. El Proceso de Formación Profesional.", en Gestión e Internacionalización de las Universidades Chilenas, CINDA-MINEDUC, 1998. 